Cahiers
de a Recherche
sur les Droits

Cahiers de la recherche sur les droits fondamentaux

15 | 2017

Le corps humain saisi par le droit : entre liberté et propriété

\title{
Le contrat de prostitution : entre ombre et lumière
}

The Contract of Prostitution: A Twilight Area

\section{Aloïse Quesne}

\section{(2) OpenEdition}

Journals

Édition électronique

URL : https://journals.openedition.org/crdf/550

DOI : $10.4000 /$ crdf.550

ISSN : 2264-1246

Éditeur

Presses universitaires de Caen

Édition imprimée

Date de publication : 1 novembre 2017

Pagination : 65-75

ISBN : 978-2-84133-858-0

ISSN : $1634-8842$

Référence électronique

Aloïse Quesne, "Le contrat de prostitution : entre ombre et lumière », Cahiers de la recherche sur les droits fondamentaux [En ligne], 15 | 2017, mis en ligne le 01 octobre 2019, consulté le 14 novembre 2022. URL : http://journals.openedition.org/crdf/550 ; DOI : https://doi.org/10.4000/crdf.550 


\title{
Le contrat de prostitution: entre ombre et lumière
}

\author{
Aloïse QUESNE \\ Doctorante en droit privé \\ Attachée temporaire d'enseignement et de recherche en droit privé à l'université de Caen Normandie \\ Institut Demolombe (EA 967)
}

I. De l'illicéité du contrat de prostitution discutée...

A. Les fondements traditionnels de cette idéologie

1. Le contrat de prostitution serait contraire au principe d'indisponibilité du corps humain

2. Le contrat de prostitution serait contraire au principe de non-patrimonialité du corps humain

3. Le consentement de la personne qui se prostitue serait vicié

4. Le contrat de prostitution serait contraire à la dignité humaine

B. Le rejet de cette idéologie

II. ...À l'illicéité du contenu du contrat de prostitution consacrée par le législateur

A. La nullité du contrat de prostitution

B. Les apories du droit face au contrat de prostitution

Entre intimité et identité. Dérivé du latin interior, l'intime désigne

[...] ce qui est au plus profond d'un être, comme le sont l'intérieur de son corps et les orifices qui font communiquer cet intérieur avec l'extérieur du monde ${ }^{1}$.

En conséquence, l'intimité touche à l'identité de l'individu. En effet, l'intimité renvoie à ce qui est invisible, tenu secret, «impénétrable et qui fonde le sentiment immédiat de son individualité propre $»^{2}$. Ainsi parle-t-on de «rapports intimes» pour désigner les rapports sexuels, lesquels appartiennent à la sphère privée.

De l'instinct de reproduction au désir. Que peut-il y avoir de plus évocateur du corps humain dans ses relations avec autrui que le rapport sexuel ? Ce contact charnel qui fusionne les corps est en effet un processus naturel en vue de la reproduction, permettant ainsi de perpétuer l'espèce. Mais les relations impliquant une union des corps sont aussi empreintes de désir. Une auteure souligne en ce sens que

L'un des domaines où se révèlent les relations complexes que chacun entretient avec son corps et avec le corps des autres est le domaine sexuel. Ne serait-ce que parce que nos relations à l'autre les plus intenses sont les relations sexuelles, et que le désir érotique est toujours une ouverture à autrui à la fois dans sa corporéité et dans sa subjectivité3

Spinoza considérait que toute chose s'efforce de persévérer dans son être. Il expliquait ainsi que

1. O. Bourguignon, "L'intime, le corps et la relation de soin ", in Traité de bioéthique, t. II, Soigner la personne, évolutions, innovations thérapeutiques, E. Hirsch (dir.), Toulouse, Érès (Espace éthique), 2010, p. 84.

2. Ibid., p. 85 .

3. M. Marzano, La philosophie du corps, Paris, PUF (Que sais-je?), 2007, p. 107. 
Cet effort, en tant qu'il a rapport à l'âme seule, s'appelle: Volonté. Mais lorsqu'il a rapport en même temps à l'Âme et au Corps, il se nomme: Appétit. L'appétit, par conséquence, n'est pas autre chose que l'essence même de l'homme [...]. En outre, entre l'appétit et le désir il n'existe aucune différence, sauf que le désir s'applique, la plupart du temps, aux hommes lorsqu'ils ont conscience de leur appétit et, par suite, le désir peut être ainsi défini: "Le désir est un appétit dont on a conscience ${ }^{4}$.

Le désir transforme alors l'union naturelle des corps permettant la reproduction de l'espèce humaine en une recherche de l'assouvissement d'une pulsion sexuelle, notion à laquelle Freud s'est particulièrement intéressée 5 . Selon l'opinion commune, les caractères de cette pulsion se manifestent

[...] sous la forme d'une attraction irrésistible exercée par l'un des sexes sur l'autre, et [...] son but serait l'union sexuelle, ou du moins un ensemble d'actes qui tendent à ce but ${ }^{6}$.

Il résulte de cette définition que le corps de l'autre est alors perçu comme un moyen de satisfaire une pulsion sexuelle et, par conséquent, la prostitution serait une activité ${ }^{7}$ qui contribuerait à l'assouvissement de ce désir sexuel.

Les multiples visages de la prostitution. De nombreux clichés circulent à propos de la prostitution. Ainsi, malgré les origines lointaines de cette pratique, la prostitution est "souvent qualifiée, à tort d'ailleurs, de plus vieux métier du monde $»^{8}$. Autre idée reçue, la prostitution serait, dans l'imaginaire collectif, l'apanage de la gent féminine. Ce préjugé est pourtant à nuancer ${ }^{9}$. La prostitution masculine existe en effet, et elle n'est d'ailleurs pas rare puisqu'elle représenterait 20 à $30 \%$ de la prostitution de rue ${ }^{10}$. Ce pourcentage ne prenant pas en compte les hommes ayant recours à Internet pour leur activité, l'estimation relative à la prostitution masculine est nécessairement à revoir à la hausse ${ }^{11}$

Le phénomène prostitutionnel saisi par le droit. Tiré du verbe latin prostituere signifiant « exposer publiquement», la prostitution serait une forme d'exhibition de ce qui relève habituellement de l'intime. Si le législateur français n'a pas donné de définition à la prostitution ${ }^{12}$, il est en revanche possible de se référer à la jurisprudence, laquelle définit la prostitution comme le fait de

[...] se prêter, moyennant rémunération, à des contacts physiques de quelque nature qu' ils soient, afin de satisfaire les besoins sexuels d'autrui ${ }^{13}$.

À la lumière de cette définition prétorienne, on comprend que c'est l'échange d'ordre pécuniaire qui constitue le principal critère de cette activité. La prostitution permet ainsi à une personne d'assouvir le désir sexuel d'autrui contre paiement. En conséquence, la tarification du rapport sexuel jette le trouble sur cette activité intime et charnelle. Une auteure reconnaît alors que,

[...] si les actes de nature sexuelle doivent assurément relever de la liberté et des choix personnels, la frontière entre la libre intimité et l'intimité contrôlée par le droit se trouble lorsqu'aux liens entre les corps s'ajoute un prix ${ }^{14}$.

4. B. Spinoza, Éthique [1677], R. Lantzenberg (trad.), Paris, Flammarion, 1947, livre III, théorèmes VI, VII et scolie du théorème IX, p. $140-142$.

5. S. Freud, Trois essais sur la sexualité [1905], B. Reverchon-Jouve (trad.), Paris, Gallimard, 1962, p. 17-18: «Pour expliquer les besoins sexuels de l'homme et de l'animal, on se sert, en biologie, de l'hypothèse qu'il existe une "pulsion sexuelle", de même que pour expliquer la faim, on suppose la pulsion de nutrition. Toutefois, le langage populaire ne connait pas de terme qui, pour le besoin sexuel, corresponde au mot faim; le langage scientifique se sert du terme: "libido"».

6. Ibid.

7. Voir A. Mazouz, Le prix du corps humain, thèse de doctorat en droit, université Paris 1 - Panthéon-Sorbonne, 2014, p. 173, $\mathrm{n}^{\circ} 358$. Selon l'auteure, l'activité peut se définir comme «la variété des manifestations de la mise en mouvement du corps, laquelle peut aussi bien intervenir à titre habituel qu'occasionnel».

8. A. Cerf-Hollender, «Libre disposition de son corps et prostitution», in La libre disposition de son corps, J.-M. Larralde (dir.), Bruxelles, Nemesis Bruylant (Droit et justice; 88), 2009, p. 309. L'auteure cite M. Costes-Péplinski, Nature, culture, guerre et prostitution: le sacrifice institutionnalisé $d u$ corps, Paris - Budapest - Turin, L'Harmattan, 2002, selon laquelle: «La première trace de vie humaine retrouvée à ce jour remonte à 6 millions d'années, le premier outil date de 2,5 millions d'années alors que la prostitution apparaît, comme la guerre, seulement à la fin du néolithique, soit 5000 ans avant Jésus-Christ au grand maximum. C'est dire si les hommes et les femmes ont partagés mille autres occupations et préoccupations avant de s'adonner à celle-ci». Voir aussi, pour une mise en perspective historique, A. Maugère, Les politiques de la prostitution: du Moyen-Âge au XXI siècle, Paris, Dalloz (Nouvelle bibliothèque de thèses), 2009.

9. Voir L. Mathieu, Sociologie de la prostitution, Paris, La Découverte (Repères. Sociologie), 2015, spéc. chap. IV: «Protagonistes du monde de la prostitution", p. 71-93.

10. Voir D. Derycke, Les politiques publiques et la prostitution. Rapport d'information sur l'activité de la délégation aux droits des femmes et à l'égalité des chances entre les hommes et les femmes pour l'année 2000, n² 209, Sénat, 31 janvier 2001, deuxième partie, "II. Panorama de la prostitution actuelle», p. 42 : «La prostitution masculine est en forte augmentation, elle atteint même 30 \% à Paris et dans les grandes agglomérations ». Voir aussi M. Olivier, Rapport d'information fait au nom de la délégation aux droits des femmes et à l'égalité des chances entre les hommes et les femmes sur le renforcement de la lutte contre le système prostitutionnel, $\mathrm{n}^{\mathrm{O}} 136 \mathrm{o}$, enregistré à la présidence de l'Assemblée nationale le 17 septembre 2013 , p. 17.

11. Les nouvelles technologies ont l'avantage de faciliter les contacts discrets avec les clients. Aussi, de plus en plus de jeunes se prostituent occasionnellement par ce biais, dans le but d'obtenir de manière rapide un complément de revenu. Pour approfondir cette forme récente de prostitution, voir E. Clouet, La prostitution étudiante à l'heure des technologies de communication, Paris, M. Milo (Essais, documents), 2008.

12. Un décret du 5 novembre 1947, qui fut ultérieurement abrogé, décrivait la prostitution comme «le fait de consentir habituellement et moyennant rémunération à des contacts sexuels avec autrui».

13. Cass. crim., 27 mars 1996, n 95-82016, Bulletin criminel, nº 138, p. 396 : Droit pénal, 1996, p. 182, obs. M. Véron. Outre le versement d'une somme d'argent, la rémunération s'entend de tout avantage matériel. Voir Cass. crim., 22 juillet 1959: Revue de sciences criminelles, 196o, p. 80, obs. L. Hugueney.

14. A. Mazouz, Le prix du corps humain, p. $247, \mathrm{n}^{\circ} 522$. 
La loi du 4 mars $2002^{15}$, celle du 18 mars $2003^{16}$ ainsi que la récente loi du 13 avril $2016^{17}$ ont toutes les trois au moins un objectif commun: celui de pénaliser les clients des personnes qui se livrent à la prostitution. Les premières lois précitées ont d'abord incriminé le fait de «solliciter, d'accepter ou d'obtenir, en échange d'une rémunération ou d'une promesse de rémunération, des relations de nature sexuelle» de la part d'une personne qui se livre à la prostitution lorsque cette personne est mineure ou lorsqu'il s'agit d'une personne qui présente une particulière vulnérabilité due à une maladie, une infirmité, une déficience physique ou psychique ou à un état de grossesse $^{18}$. Cette infraction étant toujours en vigueur, les clients encourent 3 ans d'emprisonnement et 45000 euros d'amende, voire 7 ans d'emprisonnement et 100 ooo euros lorsqu'il s'agissait d'un mineur de 15 ans ${ }^{19}$.

La loi du 13 avril 2016 visant à renforcer la lutte contre le système prostitutionnel et à accompagner les personnes prostituées a quant à elle franchi un cap en portant la création d'une contravention de $5^{\mathrm{e}}$ classe sanctionnant de manière générale toutes les personnes ayant recours à la prostitution ${ }^{20}$. La création de cette infraction s'inscrit dans la lignée de l'idéologie «néo-abolitionniste ${ }^{21}$ selon laquelle «[p]énaliser la demande permettrait de réduire l'offre de relations sexuelles tarifées ${ }^{22}$. Cette position adoptée par le législateur français pour lutter contre le phénomène prostitutionnel est en effet marquée par la volonté affirmée de ne pas sanctionner la personne qui se prostitue. L'activité prostitutionnelle n'est alors volontairement pas favorisée, et les obstacles à la liberté de son exercice se justifient par l'objectif de protection de la personne prostituée en tant que «victime à protéger des autres, mais aussi d'elle-même ${ }^{23}$. La récente abrogation du racolage par la loi du 13 avril $2016^{24}$, lequel consistait à inciter autrui à des relations sexuelles en l'échange d'une rémunération ${ }^{25}$, a contribué à renforcer cet objectif de protection. Il était en effet difficilement concevable d'envisager les mêmes personnes comme victimes et délinquantes à la fois.

Enjeux et perspectives du contrat de prostitution. À l'occasion d'un article consacré à la vente du corps humain publié en 2011, le professeur Gérard Mémeteau s'interrogeait à propos de l'activité prostitutionnelle, se demandant si l'on pouvait réellement parler de contrat de prostitution. Cet auteur posait ainsi la question suivante: "Quelles obligations naitraient-elles du contrat d'intimité? $»^{26}$. Ces interrogations étaient révélatrices de l'attitude des juristes qui envisageaient surtout le fait prostitutionnel en dehors du droit. Depuis lors, une thèse récente ${ }^{27}$ a relevé

15. Loi n ${ }^{\circ}$ 2002-305 du 4 mars 2002 relative à l'autorité parentale.

16. Loi $\mathrm{n}^{\mathrm{o}} 2003-239$ du 18 mars 2003 pour la sécurité intérieure.

17. Loi no 2016-444 du 13 avril 2016 visant à renforcer la lutte contre le système prostitutionnel et à accompagner les personnes prostituées, Journal officiel de la République française, $\mathrm{n}^{\circ} \mathrm{0o88}, 14$ avril 2016, texte $\mathrm{n}^{\circ} 1$. Sur laquelle, voir N. Laurent-Bonne, «La lutte contre le système prostitutionnel. Analyse critique et comparative de la loi n² 2016-444 du 13 avril 2016", Recueil Dalloz, 2016, p. 1713 sq.; A. Casado, «Brèves remarques à la lecture de la loi n' 2016-444 du 13 avril 2016 visant à renforcer la lutte contre le système prostitutionnel et à accompagner les personnes prostituées", Droit pénal, $\mathrm{n}^{\circ}$ 6, 2016, étude 12.

18. Ancien art. L. 225-12-1 du Code pénal (loi no 2002-305 du 4 mars 2002, art. 13; loi no 2003-239 du 18 mars 2003, art. 50). Incrimination aujourd'hui prévue à l'article L. 225-12-1, alinéa 2 du Code pénal (loi n 2016-444 du 13 avril 2016, art. 20). Le terme de «handicap» a remplacé la « déficience physique ou psychique».

19. Art. L. 225-12-2 $4^{\circ}$ du Code pénal.

20. Le chapitre V de la loi n 2016-444 du 13 avril 2016 s'intitule «Interdiction de l'achat d'un acte sexuel». L'article 20 de cette même loi prévoit la création d'un titre unique inséré au livre VI du Code pénal, lequel a pour titre «Du recours à la prostitution». L'infraction est alors consacrée au nouvel article 611-1 du Code pénal.

21. Parmi les actions abolitionnistes, la loi Marthe Richard du 13 avril 1946 a abrogé les dispositions qui prévoyaient l'inscription des personnes qui se livraient à la prostitution sur des fichiers de police spéciaux et fermé les maisons closes. Il n'existe pas de consensus des États en ce qui concerne la prostitution. Certains États choisissent d'adopter un régime dit réglementariste qui permet d'encadrer l'activité prostitutionnelle (c'est le cas des Pays-Bas notamment), d'autres États dits prohibitionnistes interdisent la prostitution (comme la Chine et bon nombre d'États américains par exemple), enfin, des systèmes mixtes dits de «néo-abolitionnisme» n'incriminent pas la prostitution mais sanctionnent les clients qui y ont recourt, c'est la position adoptée par la Suède et depuis peu par la France.

22. N. Laurent-Bonne, «La lutte contre le système prostitutionnel... », \$2.

23. A. Mazouz, Le prix du corps humain, p. $256, \mathrm{n}^{\circ} 543$.

24. Loi n' 2016-444 du 13 avril 2016, art. 15.

25. L'ancien article 225-10-1 du Code pénal incriminait le racolage public, lequel était défini comme «le fait, par tout moyen, y compris par une attitude même passive, de procéder publiquement au racolage d'autrui en vue de l'inciter à des relations sexuelles en échange d'une rémunération ou d'une promesse de rémunération». Cette infraction était punie de 2 mois d'emprisonnement et de 3750 euros d'amende. Voir A. Cerf-Hollender, «Libre disposition de son corps et prostitution", p. 321, où l'auteure explique qu'à l'origine le racolage était passible d'une contravention de $3^{\mathrm{e}}$ classe créée en 1939, puis correctionnalisée en 1946. L'ordonnance du 23 décembre 1958 a remplacé le délit par deux contraventions, de $3^{\mathrm{e}}$ classe pour racolage actif et de $1^{\text {re }}$ classe pour racolage passif, lesquelles sont devenues de $5^{\mathrm{e}}$ et de $3^{\mathrm{e}}$ classe par une ordonnance du 25 novembre 1960 . Puis le Code pénal de 1992 avait abrogé le racolage passif, pour ne maintenir que la contravention de $5^{\mathrm{e}}$ classe pour le racolage actif (art. R. 625-8). Mais la loi du 18 mars 2003 relative à la sécurité intérieure avait étendu le champ d'application de la répression, n'opérant plus de distinction entre le racolage actif et le racolage passif, aggravant par la même occasion la répression du racolage, passant d'une infraction contraventionnelle à un délit correctionnel. La Cour de cassation a eu l'occasion de se prononcer à propos du racolage passif, lequel supposait, au titre de son élément matériel, une action entreprise par la personne qui se prostitue pour racoler autrui. La Cour avait ainsi estimé que le fait de stationner en un lieu connu pour la prostitution dans une tenue provocante n'était pas constitutif de cette infraction (Cass. crim., 25 mai 2005, $\mathrm{n}^{\circ}$ 04-84-769: Droit pénal, $\mathrm{n}^{\circ} 10,2005$, comm. 138, obs. M. Véron). L'absence d'indication précise relative à l'action entreprise par la personne rendait parfois difficile une telle constatation.

26. G. Mémeteau, «La vente du corps humain », Les cahiers de droit de la santé du Sud-Est: juridiques, historiques et prospectifs, $\mathrm{n}^{\circ}$ 12, 2011, Les éléments et produits du corps humain, G. Nicolas (dir.), p. 27-48, spéc. p. 41.

27. A. Casado, La prostitution en droit français: étude de droit privé, préface de G. Loiseau, Paris, IRJS (Bibliothèque IRJS - André Tunc; 62), 2015 
le défi d'éprouver les faits de la prostitution sous l'angle du droit des contrats, à savoir: un accord de volonté entre deux personnes sur un objet qui ne serait pas le corps de la personne faisant commerce de ses charmes, mais la réalisation d'une prestation de service sexuel.

Le contrat de prostitution, en tant qu'il implique la mise en jeu du corps humain contre rémunération en vue de satisfaire les besoins sexuels d'autrui, met à l'épreuve les valeurs et principes cardinaux de notre droit. Le législateur, en prohibant l'achat d'actes sexuels, met aujourd'hui tout en ouvre pour neutraliser ce contrat. Cette nouvelle ère impose le constat suivant: nous sommes en train d'assister au passage de l'illicéité du contrat de prostitution discutée (I), à l'illicéité du contenu du contrat de prostitution consacrée par le législateur (II).

\section{De l'illicéité du contrat de prostitution discutée...}

La prostitution au cour d'un débat sociétal. Les partisans de la doctrine de victimisation affirment fermement que le contrat de prostitution ne répond pas aux conditions de validité du droit commun des contrats. Seront ainsi examinés les fondements de cette idéologie (A). Pour contourner l'interdit, la jurisprudence européenne, suivie par une partie de la doctrine, a rejeté cette idéologie, préférant regarder le contrat de prostitution comme portant sur une prestation de service sexuel (B).

\section{A. Les fondements traditionnels de cette idéologie}

\section{Le contrat de prostitution serait contraire au principe d'indisponibilité du corps humain}

Indisponibilité et inviolabilité du corps humain. La loi n 94-653 du 29 juillet 1994 n'a pas expressément inscrit le principe d'indisponibilité du corps humain dans le Code civil. Le législateur a choisi d'inscrire, aux termes de l'article 16-1 du Code civil ${ }^{28}$, le principe de l'inviolabilité du corps humain plutôt que celui de l'indisponibilité. Certains auteurs voient cependant, à travers l'inscription du principe d'inviolabilité, la consécration du principe d'indisponibilité du corps de la personne. Cela s'explique par le fait que ces auteurs considèrent que l'inviolabilité du corps, si elle s'applique à autrui, s'applique également à la personne ${ }^{29}$. Mais le principe d'inviolabilité consiste en l'interdiction qui est faite à autrui de porter atteinte à l'intégrité corporelle d'une personne ${ }^{30}$ sans son consentement, ce qui démontre bien que le législateur avait l'intention de conférer à la personne un certain pouvoir sur son corps.

Disponibilité du corps humain en matière sexuelle. Le pouvoir de disposer de son corps est appuyé par le droit européen, lequel reconnaît une liberté sexuelle fondée sur le consentement des personnes. Dans l'affaire Pretty c. Royaume-Uni ${ }^{31}$, la Cour européenne des droits de l'homme a ainsi énoncé que l'article 8 de la Convention européenne des droits de l'homme, relatif à la vie privée, comportait un droit à l'autodétermination au sens de la possibilité d'opérer des choix concernant son propre corps $^{32}$. À l'occasion de l'affaire K. A. et A. D. c. Belgique $^{33}$, laquelle traitait de relations sadomasochistes librement consenties, la même juridiction a considéré que le droit d'entretenir des relations sexuelles découle du droit de disposer de son corps, qui fait partie intégrante de la notion d'autonomie personnelle ${ }^{34}$. Si les relations sexuelles librement consenties relèvent de la sphère privée, le droit ne devrait en principe pas intervenir en ce qui concerne la prostitution. Mais la question ne va pas nécessairement de soi, car cet acte onéreux à l'encontre du corps humain est régulièrement invoqué comme étant contraire au principe de non-patrimonialité du corps humain.

\section{Le contrat de prostitution serait contraire au principe de non-patrimonialité du corps humain}

Définition du principe de non-patrimonialité du corps humain. La non-patrimonialité du corps humain est posée à l'article 16-1, alinéa 3, du Code civil qui prévoit que: «Le corps humain, ses éléments et ses produits ne peuvent faire l'objet d'un droit patrimonial». Cela signifie que le corps humain est «en dehors du patrimoine, intéressant la personne elle-même et non ses biens ${ }^{35}$. Le corps humain ne peut alors faire l'objet que d'un droit extrapatrimonial, c'est-à-dire d'un droit "présentant pour le sujet un intérêt moral, n'ayant aucun

28. Art. 16-1 du Code civil: «Le corps humain est inviolable./Le corps humain, ses éléments et ses produits ne peuvent faire l'objet d'un droit patrimonial».

29. Voir M.-J. Redor-Fichot, «Rapport de synthèse», in La libre disposition de son corps, p. 341

30. Vocabulaire juridique, $9^{\mathrm{e}}$ éd., G. Cornu (dir), Paris, PUF, 2009, entrée «Inviolabilité».

31. Cour EDH, 29 avril 2002, Pretty c. Royaume-Uni, n' 2346/o2.

32. Pour ce faire, la Cour européenne des droits de l'homme s'est fondée sur la notion «d'autonomie personnelle». Elle a ainsi énoncé que «bien qu'il n'ait pas été établi dans aucune affaire antérieure que l'article 8 de la Convention comporte un droit à l'autodétermination en tant que tel, la Cour considère que la notion d'autonomie personnelle reflète un principe important qui sous-tend l'interprétation des garanties de l'article 8 " (\$61). Le droit à la vie privée comporte ainsi un droit à l'autodétermination "au sens du choix d'opérer des choix concernant son propre corps» $(\$ 66)$.

33. Cour EDH, 17 février 2005, K. A. et A. D. c. Belgique, nº 42758/98 et 45558/99: M. Fabre-Magnan, "Le sadisme n'est pas un droit de l'homme», Recueil Dalloz, 2005, p. 2973 sq.

34. Cour EDH, 17 février 2005, K. A. et A. D. c. Belgique, $\$ 83$.

35. Lexique des termes juridiques, S. Guinchard, G. Montagnier (dir.), 17 éd., Paris, Dalloz, 20o9, entrée «Extrapatrimonial». 
contenu pécuniaire et ne représentant pour son titulaire aucun élément de richesse matérielle ${ }^{36}$. L'article 16-5 dispose ensuite que: «Les conventions ayant pour effet de conférer une valeur patrimoniale au corps humain, à ses éléments ou à ses produits sont nulles». La valeur patrimoniale peut s'analyser comme le critère de distinction entre le patrimonial et l'extrapatrimonial, la valeur patrimoniale désigne alors l'estimation d'une chose ${ }^{37}$. En posant l'interdit d'évaluer le corps humain à l'aune d'une somme d'argent, le législateur entend maintenir l'impossibilité de faire entrer le corps humain dans le patrimoine de la personne ${ }^{38}$. Le corps humain, s'il est dans le commerce juridique, est en principe exclu du marché ${ }^{39}$.

Le principe de non-patrimonialité à l'épreuve de la prostitution. Si certains auteurs considèrent que la prostitution peut être assimilée à la vente ${ }^{40}$ ou à la location ${ }^{41}$ du corps de la personne, une partie de la doctrine s'accorde à dire que «la prostitution donne au corps humain une valeur pécuniaire $»^{42}$ puisque le corps est mis à disposition d'autrui en l'échange d'une rémunération, laquelle entre dans le patrimoine de la personne qui se prostitue.
La prostitution serait donc contraire au principe de nonpatrimonialité en ce que cette activité fait du corps humain une source de profit ${ }^{43}$.

\section{Le consentement de la personne qui se prostitue serait vicié}

La liberté du consentement mise à mal. Le contrat de prostitution implique nécessairement le consentement de la personne qui se prostitue. Son consentement est protégé par l'article 222-23 du Code pénal puisque tout acte de pénétration sexuelle commis sur la personne d'autrui sans son consentement est considéré comme un viol $^{44}$. Mais la difficulté porterait sur le caractère libre et éclairé du consentement donné. Ceux qui voient dans la prostitution un contrat qui ne saurait reposer sur un consentement lucide et éclairé estiment que les femmes sont davantage visées par ce phénomène et qu'elles se trouvent dans un état de grande vulnérabilité sociale qui les poussent à se prostituer. Leur vulnérabilité pèserait alors sur la liberté de leur consentement, ce qui revient à dire qu'il n'y aurait jamais vraiment de prostitution volontaire ${ }^{45}$.

36. A. Montas, «Entre "être" et "avoir", le corps humain est-il vénal?», Revue de la recherche juridique. Droit prospectif, n 4, 2006 , p. 2245.

37. Voir J.-C. Galloux, «De corpore jus, premières analyses sur le statut juridique du corps humain, ses éléments et ses produits selon les lois $\mathrm{n}^{\circ} 94-653$ et 94-654 du 29 juillet 1994 ", Petites affiches, nº 149, 14 décembre 1994.

38. Cependant, le législateur autorise de façon limitée le corps humain à intégrer d'autres patrimoines. À titre d'exemple, en ce qui concerne le don d'éléments et produits du corps humain, ces derniers, une fois prélevés, intègrent le patrimoine des établissements en charge de leur prélèvement ou de leur collecte. Voir C. Labrusse-Riou, «Difficultés, contradictions et apories du droit de la "bioéthique” ", in Le droit privé français à la fin $d u X^{e}$ siècle. Études offertes à Pierre Catala, Paris, Litec, 2001, p. 275-289.

39. Voir G. Loiseau, «Typologie des choses hors du commerce», Revue trimestrielle de droit civil, 200o, spéc. p. 53. Certains produits du corps humain peuvent par exception faire l'objet de conventions à titre onéreux selon l'article L. 1211-8 du Code de la santé publique. Il s'agit des phanères. Ces produits, déterminés par décret, sont: les dents, les cheveux, les ongles et les poils. La justification de leur vénalité parait critiquable.

40. Un auteur a notamment ouvert deux hypothèses en ce sens: celle de la vente à temps et celle de la multipropriété du corps de la personne qui se prostitue. Voir C. Amourette, Prostitution et proxénétisme en France depuis 1946 : étude juridique et systémique, thèse de doctorat en histoire du droit, université Montpellier 1, 2003, spéc. p. 239 sq. Contra, A. Casado, La prostitution en droit français..., spéc. $\mathrm{n}^{\circ} 895$ à 897, qui réfute la qualification de contrat de vente et notamment à travers la notion de transfert de propriété qui fait défaut.

41. En ce sens, voir le philosophe E. Kant, Leçons d'éthique, L. Langlois (éd. et trad.), Paris, Librairie générale française (Classiques de la philosophie), 1997, p. 293, selon qui: "Il n'y a rien de plus honteux que d'offrir ainsi sa personne en location et de la livrer pour de l'argent à la satisfaction de l'inclination sexuelle d'un autre». Voir N. Campagna, Prostitution et dignité, Paris, La Musardine (L'attrape-corps), 2008, p. 95; cet auteur explique que la personne qui se livre à la prostitution «loue son vagin, sa bouche, ses mains ou encore d'autres parties de son corps». Contra, A. Casado, La prostitution en droit français..., spéc. no 902 à 905 , lequel réfute la qualification du contrat de louage.

42. L. Ouvrard, La prostitution. Analyse juridique et choix de politique criminelle, Paris - Montréal, L'Harmattan, 2000, p. 151.

43. Voir les propositions de loi $n^{\circ} 1436$ et $n^{\circ} 1347$, renforçant la lutte contre le système prostitutionnel, enregistrées à la présidence de l'Assemblée nationale le 10 octobre 2013, qui précisent que la résolution parlementaire $\mathrm{n}^{\circ} 3522 \mathrm{du} 6$ décembre 2011 va dans ce sens en rappelant que la prostitution «heurt[e] les principes fondamentaux de notre société [...]. La non-patrimonialité du corps humain, l'un des principes cardinaux de notre droit, fait obstacle à ce que le corps humain soit considéré comme une source de profit».

44. Art. 222-23 du Code pénal: «Tout acte de pénétration sexuelle, de quelque nature qu'il soit, commis sur la personne d'autrui par violence, contrainte, menace ou surprise est un viol».

45. Dans le cadre de la Commission d'information parlementaire sur la prostitution, $\mathrm{M}^{\mathrm{me}}$ la ministre Roselyne Bachelot-Narquin, ministre des Solidarités et de la Cohésion sociale, est intervenue en audition publique le 30 mars 2011 en ces termes: «Je veux le dire, ici sans ambiguïté, contrairement à ce que certains voudraient nous faire croire, il n'existe pas de prostitution libre, choisie ou consentie. Par définition la prostitution est toujours une violence faite aux femmes et d'abord aux plus vulnérables d'entre elles, celles qui se trouvent dans une situation de grande précarité». Contra, certaines personnes se revendiquent des «travailleurs du sexe» et militent pour une reconnaissance officielle de cette profession, considérant que la prostitution libre existe. La revendication d'une prostitution choisie et reconnue appartient même désormais à une nouvelle branche du combat féministe. Sur ce phénomène, voir L. Starck, «Néo-féminisme et prostitution», in Le sexe et la norme, N. Deffains, B. Py (dir.), Nancy, Presses universitaires de Nancy (Santé, qualité de vie et handicap), 2011, p. 397 sq. Dans le même sens, voir A. Cerf-Hollender, «Libre disposition de son corps et prostitution", p. 314, laquelle cite une lettre ouverte qui avait été adressée à Noël Mamère par le collectif Droits et prostitution : "Oui, nous avons des tas de problèmes et des parcours souvent difficiles étant le plus souvent des femmes ou des minorités déjà discriminées. Mais le travail du sexe a pu représenter au cours de nos vies une ressource, afin de migrer, d'étudier, de veiller au bien de sa famille et de ses enfants, d'être indépendant économiquement, bref de progresser socialement. Cela ne plaît peut-être pas que nous utilisions notre sexe, mais l'usage de son sexe ne veut pas dire que l'on perd l'usage de son cerveau». Voir aussi Maîtresse Nikita, T. Schaffauser, Fières d'être putes, Castres, L'Altiplano (Agit'Prop), 2007. 


\section{Le contrat de prostitution serait contraire à la dignité humaine}

Éléments de définition de la dignité humaine. La dignité 46 constitue la matrice de notre système juridique ${ }^{47}$. Pour tenter d'apporter une définition de la dignité, nous pouvons reprendre la formule d'un auteur selon laquelle «la dignité correspond à l'essence de l'homme, c'est ce qui permet de distinguer l'homme de l'animal et des choses en général ${ }^{48}$. Il est classique de dire que «la définition de la dignité est délicate et son contenu relève d'une interprétation casuistique et évolutive ${ }^{49}$. Dans une conception universaliste, la dignité représente un «impératif universel permettant d'assurer la protection minimale à laquelle toute personne a droit en tant qu'être humain $»^{50}$. C'est en ce sens que cette acception «fait de la dignité une qualité opposable à l'homme par des tiers ${ }^{51}$ et que le consentement n'est pas toujours suffisant pour rendre licite un acte, comme en témoigne la décision prise par les juges administratifs dans la célèbre jurisprudence Commune de Morsang-sur$\mathrm{Orge}^{52}$. En l'espèce, les juges ont considéré que la dignité humaine était une composante de l'ordre public, ce qui rendait légale l'interdiction par le maire de la commune de procéder à l'activité de "lancer de nain", et ce même en l'absence de circonstances locales particulières. Alors même que l'intéressé avait consenti à ce qu'on l'utilise comme un projectile, que cette activité était dépourvue de risque pour sa santé puisqu'il bénéficiait d'un harnachement spécifique destiné à prévenir d'éventuelles lésions corporelles, et que cette activité était importante pour lui car elle lui permettait de subvenir à ses besoins, une telle activité a été déclarée illicite, car contraire à la dignité de la personne humaine.

Compatibilité de la prostitution avec la dignité. Le droit européen, s'il a pris position de façon claire en ce qui concerne la liberté sexuelle, ne s'est en revanche pas prononcé aussi franchement en ce qui concerne la prostitution. Ainsi, dans l'affaire Tremblay c. France ${ }^{53}$, la juridiction européenne a d'abord constaté «qu'il est manifeste qu'il n'y a pas de consensus européen quant à la qualification de la prostitution en elle-même " ${ }^{54}$, avant de préciser «qu'elle juge la prostitution incompatible avec les droits et la dignité de la personne humaine dès lors qu'elle est contrainte ${ }^{55}$. Le préambule de la Convention des Nations unies pour la répression de la traite des êtres humains et de l'exploitation de la prostitution d'autrui du 2 décembre 1949 énonce quant à lui clairement que

[...] la prostitution et [...] la traite des êtres humains en vue de la prostitution sont incompatibles avec la dignité et la valeur de la personne humaine ${ }^{56}$.

La ratification de cette Convention a permis d'inscrire le choix de la politique abolitionniste fait par la France, en affirmant que l'activité prostitutionnelle est contraire aux valeurs qu'elle défend. Une partie de la doctrine considère dans le même sens que la dignité doit avant tout être envisagée en tant que composante de l'humanité qui se situe, comme le corps humain, hors commerce. Or, la prostitution donnant au corps humain une valeur pécuniaire, la personne prostituée se situerait, de fait, au sein d'une exploitation commerciale ${ }^{57}$.

46. Sur laquelle, voir notamment B. Mathieu, «La dignité de la personne humaine: du bon (et du mauvais?) usage en droit positif français d'un principe universel ", in Le droit, la médecine et l'être humain. Propos hétérodoxes sur quelques enjeux vitaux du XXI siècle, A. Sériaux et al. (dir.), Aix-en-Provence, Presses universitaires d'Aix-Marseille, 1996, p. 213-236; La dignité de la personne humaine, M.-L. Pavia, T. Revet (dir.), Paris, Economica (Études juridiques; 7), 1999; Éthique, droit et dignité de la personne humaine. Mélanges Christian Bolze, P. Pedrot (dir.), Paris, Economica, 1999; X. Bioy, Le concept de personne humaine en droit public. Recherche sur le sujet des droits fondamentaux, Paris, Dalloz (Nouvelle bibliothèque de thèses; 22), 2003; M. Fabre-Magnan, «La dignité en droit: un axiome», Revue interdisciplinaire d'études juridiques, $\mathrm{n}^{\circ}$ 58, 2007, p. 1-30; J.-P. Feldman, «Faut-il protéger l'homme contre lui-même? La dignité de l'individu et la personne humaine», Droits, no 48 , 2009, p. 87-108. Pour un exposé des «origines du principe de respect de la dignité de la personne humaine», voir M. Mekki, L'intérêt général et le contrat: contribution à une étude de la hiérarchie des intérêts en droit privé, Paris, LGDJ (Bibliothèque de droit privé), 2004, spéc. ${ }^{\circ}{ }^{426}$ sq.

47. Voir J.-M. Verdier, «Le droit du travail, terre d'élection pour les droits de l'homme», in Les orientations sociales du droit contemporain. Écrits en l'honneur du professeur Jean Savatier, P. Couvrat (dir.), Paris, PUF, 1992, p. 431. L'auteur y affirme que «la dignité de la personne humaine constitue le concept central et le fondement de la théorie des droits de l'homme et se trouve affirmée, avant même les droits garantis, dans presque tous les instruments internationaux récents relatifs aux droits de l'homme [...] et fonde la philosophie de la Convention européenne [...]. Elle est aussi au cœur de grandes conceptions, philosophiques ou religieuses, en matière de droits de l'homme [...]. Elle fournit l'idée, force de la doctrine juridique en ce domaine».

48. E. Dreyer, «La dignité opposée à la personne», Recueil Dalloz, 2008, p. 2730.

49. M. Fabre-Magnan, «Dignité», in Dictionnaire des droits de l’homme, J. Andriantsimbazovina et al. (dir.), Paris, PUF, 2008, p. 227.

50. E. Dreyer, «Les mutations du concept juridique de dignité», Revue de la recherche juridique. Droit prospectif, $\mathrm{n}^{\circ} 1,2005, \mathrm{p} .19$.

51. La dignité de la personne humaine: recherche sur un processus de juridicisation, C. Girard, S. Hennette-Vauchez (dir.), Paris, PUF (Droit et justice), 2005 , p. 24.

52. CE, 27 octobre 1995, Commune de Morsang-sur-Orge, Recueil Lebon, p. 372.

53. Cour EDH, 11 décembre 2007, Tremblay c. France, $\mathrm{n}^{\circ}$ 37194/02: Revue trimestrielle de droit civil, 2007, p. 730, note J.-P. Marguénaud.

54. Cour EDH, 11 décembre 2007, Tremblay c. France, $\$ 24$.

55. Ibid., $\$ 25$.

56. Convention entrée en vigueur en France le 19 novembre 1960. Voir la loi no $60-754$ du 28 juillet 1960 autorisant la ratification de la convention, Journal officiel de la République française, 30 juillet 1960, p. 7041; décret no 60-1251 du 25 novembre 1960 portant publication de la Convention pour la répression de la traite des êtres humains et de l'exploitation de la prostitution d'autrui adoptée par l'Assemblée générale des Nations unies le 2 décembre 1949, Journal officiel de la République française, 27 novembre 1960, p. 10619-10621, rectificatif Journal officiel de la République française, 15 décembre 1960, p. 11225.

57. Voir L. Ouvrard, La prostitution..., p. 151. Selon cette auteure, «la dignité doit avant tout être envisagée en tant que composante de l'humanité qui se situe, comme le corps humain, hors commerce. Or la prostitution donne au corps humain une valeur pécuniaire. La personne prostituée accepte 
Dignité, prostitution et gestation pour autrui. Mettant à l'épreuve la dignité de la personne humaine, le contrat de prostitution permet de soutenir la comparaison avec le contrat de gestation pour autrui ${ }^{5}$. L'activité de gestation pour autrui nécessite de mettre son corps à disposition d'autrui en vue de remettre au couple commanditaire l'enfant qui naîtra de cette gestation. On entend fréquemment l'expression «location d'utérus » lorsque l'on parle de la gestation pour autrui. Les termes sont critiquables: l'utérus n'étant pas un élément détachable du corps de la femme, il paraît évident que la gestation pour autrui implique une emprise sur tout le corps, quel que soit le moment ou les procédés auxquels on s'attache (stimulation ovarienne, grossesse, accouchement...). À titre de comparaison, le contrat de prostitution pourrait-il lui aussi n'être qu'une «location des organes sexuels » ? Cela est difficilement concevable. L'implication du corps est certes beaucoup moins grande dans le cadre du contrat de prostitution que dans le cadre du contrat de gestation pour autrui, mais il s'agit bien du corps entier de la personne qui est mis à disposition et pas seulement de ses organes sexuels.

Outre la question de la mise à disposition du corps, les similitudes entre ces deux contrats portent également sur la satisfaction du désir d'autrui. Satisfaction du désir d'enfant ou de parenté dans le cadre de la gestation pour autrui, et satisfaction du désir sexuel dans le cadre de la prostitution. L'exploitation du corps comme un moyen pour satisfaire le désir d'autrui est souvent en lien direct avec la misère absolue dans laquelle se trouvent certaines personnes.

Quand le corps peut constituer une source de revenus, le recours à sa marchandisation est souvent ce qu'il reste à ceux qui n'ont plus rien. Et c'est en cela que l'utilisation du corps peut s'avérer humiliante, dégradante, avilissante. Traiter le corps comme une machine à reproduction ou un objet sexuel, voilà ce qui peut conduire à considérer ces contrats comme contraires à la dignité de la personne humaine ${ }^{59}$.

\section{B. Le rejet de cette idéologie}

Une prestation de service sexuel. La jurisprudence européenne tend à considérer la prostitution comme une prestation de service rémunérée, comme en témoigne l'arrêt Jany rendu par la Cour de justice des Communautés européennes (CJCE) le 20 novembre $2001^{60}$, aux termes duquel la prostitution est

[...] une activité par laquelle le prestataire satisfait, à titre onéreux, une demande du bénéficiaire, sans produire ou céder des biens matériels [et qui] constitue une prestation de service rémunérée qui [...] relève de la notion d' $\mathrm{d}^{\text {acti- }}$ vités économiques ${ }^{61}$.

Cette terminologie s'inscrit en droit interne dans la mouvance du droit des contrats, lequel a en partie été remanié par l'ordonnance du 10 février $2016^{62}$ portant réforme du droit des contrats, entrée en vigueur le $1^{\text {er }}$ octobre 2016. En effet, l'alinéa $1^{\text {er }}$ de l'article 1163 nouveau du Code civil dispose que « [1]'obligation a pour objet une prestation présente ou future».

Contrat de prostitution et louage d'ouvrage. Une partie de la doctrine partage la position adoptée par le droit européen, en retenant la qualification de louage d'ouvrage ${ }^{63}$. Ce contrat est défini à l'article $1710 \mathrm{du}$ Code civil comme « un contrat par lequel l'une des parties s'engage à faire quelque chose pour l'autre, moyennant un prix convenu entre elles ». Le louage de service étant une des espèces principales du louage d'ouvrage selon l'article $1779 \mathrm{du}$ Code civil, la personne qui se prostitue engagerait donc ses services pour une entreprise déterminée, la fourniture d'actes sexuels contre rémunération ${ }^{64}$.

Critique de la qualification. Si la qualification retenue présente un certain intérêt en ce qu'elle permet de contourner l'interdit en écartant la personne et son corps de l'objet du contrat de prostitution, cette définition peine à convaincre complètement car elle tend à réduire, voire à nier l'étendue

(et supporte) l'humiliation de son activité en échange d'une prestation monétaire. Elle se situe, de fait, au sein d'une exploitation commerciale sous l'empire des proxénètes et confortée par la demande des clients".

58. Voir M. Fabre-Magnan, La gestation pour autrui: fictions et réalité, Paris, Fayard, 2013, spéc. p. 93-96, l'auteure soulignant que «l'analyse juridique de la nature de l'activité promise par le contrat de gestation pour autrui permet de poursuivre la comparaison avec la prostitution. La structure des obligations nées du contrat de prostitution ou du contrat de mère porteuse ne diffère pas fondamentalement de celle découlant du contrat de travail. Tous ces contrats mettent en jeu comme on l'a vu le corps d'une personne. [...] En réalité, la gestation pour autrui, comme la prostitution, $[\ldots]$ n'est certainement pas un travail comme un autre, contrairement à des propos qui ont pu être tenus et qui voudraient nous convaincre de banaliser cette pratique».

59. Voir L. Ouvrard, La prostitution..., p. 154: «La convention qui se crée entre la personne prostituée et le client porte bien sur le corps, ou tout au moins sur une partie qui n'en est pas détachable. Les seuls exemples que le droit connaisse en matière d'utilisation du corps humain dans son entier sont ceux de la maternité de substitution et celui du "lancer de nain" et il les a condamnés".

60. CJCE, 20 novembre 2001, Aldona Malgorzata Jany et autres c. Pays-Bas, C-268/99: S. Retterer, "L'activité de prostitution exercée à titre indépendant: une activité économique au sens du droit communautaire», Recueil Dalloz, 2002, p. 2144.

61. CJCE, 20 novembre 2001, Aldona Malgorzata Jany et autres $c$. Pays-Bas, $\$$ 48-49.

62. Ordonnance $\mathrm{n}^{\circ}$ 2016-131 du 10 février 2016 portant réforme du droit des contrats, du régime général et de la preuve des obligations.

63. Qualification notamment retenue par A. Casado, La prostitution en droit français..., nº 9o6. Dans le même sens, voir F. Caballero, Droit du sexe, Paris, LGDJ, 2010, $\mathrm{n}^{\circ} 527$, où l'auteur considère que «l'objet du contrat de prostitution consiste en la fourniture à autrui d'un service sexuel rémunéré».

64. Voir A. Casado, La prostitution en droit français..., $\mathrm{n}^{\circ}$ 905: «Dans le contrat de prostitution, une personne - celle qui se prostitue - engage ses services pour une entreprise déterminée - la fourniture d'actes de nature ou à connotations sexuelles contre rémunération ". 
de l'implication du corps humain. En effet, s'il y a bien prestation de service par la fourniture d'une satisfaction du corps du client, cette dernière se réalise par l'usage d'un corps déconnecté de la personnalité de celui qui se livre à la prostitution.

C'est en cela que cette prestation de service se révèle bien particulière. Si l'illicéité de la prestation de service au cœur du contrat de prostitution était jusqu'à présent en débat, le législateur a aujourd'hui permis sa consécration.

\section{II. ...À l'illicéité du contenu du contrat de prostitution consacrée par le législateur}

Le législateur face au contrat de prostitution. L'étude des dispositions de la loi du 13 avril 2016 visant à renforcer la lutte contre le système prostitutionnel et à accompagner les personnes prostituées démontre que le contrat de prostitution est désormais frappé de nullité (A). Bien que le contenu du contrat de prostitution soit désormais contraire à l'ordre public, le droit ne le prive pourtant pas de tout effet, ce qui nourrit l'analyse critique du droit positif (B).

\section{A. La nullité du contrat de prostitution}

Des entraves à la neutralisation du contrat de prostitution. Auparavant, le législateur avait mis en place des obstacles à la conclusion de ce contrat à travers le délit de racolage ${ }^{65}$, ainsi que des obstacles à sa mise en œuvre, notamment avec l'incrimination de proxénétisme. Le proxénétisme n'est pas défini par le législateur. Cette incrimination, prévue à l'article 225-5 du Code pénal, revêt plusieurs formes:

- le proxénétisme par aide, assistance ou protection de la prostitution d'autrui;

- le proxénétisme qui consiste à tirer profit de la prostitution d'autrui, à en partager les produits ou à recevoir des subsides d'une personne qui se livre à la prostitution de manière habituelle;

- et le proxénétisme qui consiste à embaucher, entraîner ou détourner une personne afin qu'elle se prostitue ou exercer sur elle une pression pour qu'elle se prostitue ou continue à le faire.
Le législateur assimile également au proxénétisme d'autres comportements qui gravitent autour de l'activité prostitutionnelle ${ }^{66}$ et qui peuvent pourtant servir de protection et d'assistance à la personne qui se prostitue. À ce titre, on peut citer le proxénétisme par fourniture de locaux, incriminé à l'article 225-10 du Code pénal ${ }^{67}$. La prostitution n'était donc ni interdite ni réglementée en tant que telle. Elle était tolérée par l'État français qui adoptait une attitude mitigée face au phénomène prostitutionnel. Mais l'attitude du législateur a désormais pris un tournant prohibitionniste par l'adoption de la loi du 13 avril 2016. Si la prostitution non contrainte relevait de la liberté entre adultes selon le droit européen ${ }^{68}$, la loi fixe désormais une limite à cette liberté sexuelle en créant le statut de victimes de la prostitution.

La création du statut de victimes de la prostitution. Aux côtés des victimes du proxénétisme et de la traite des êtres humains, le nouveau statut de victimes de la prostitution ${ }^{69}$ implique que le consentement de ces personnes puisse être considéré comme vicié, notamment d'un vice de violence. Cela marque une certaine victoire des partisans de la doctrine de victimisation car, même si l'on tient volontairement à l'écart la crainte de violences physiques, la vulnérabilité induite par ce statut et la contrainte économique qui en résulte pourraient par conséquent rendre nul leur consentement suivant l'article 1143 nouveau du Code civil, en vertu duquel:

Il y a également violence lorsqu'une partie, abusant de l'état de dépendance dans lequel se trouve son cocontractant, obtient de lui un engagement qu'il n'aurait pas souscrit en l'absence d'une telle contrainte et en tire un avantage manifestement excessif.

Un raisonnement par analogie nous permet de considérer que le client, profitant de l'état de dépendance économique dans lequel se trouve la personne prostituée, obtient de sa part l'engagement de l'accomplissement d'un acte sexuel qu'elle n'aurait pas consenti en l'absence d'une telle contrainte. L'acte sexuel ainsi obtenu représente un avantage manifestement excessif.

Interdiction de l'achat d'un acte sexuel. La loi du 13 avril 2016 porte également création de l'infraction de l'achat d'un acte sexuel ${ }^{70}$. Il est à noter que la terminologie employée par le législateur paraît peu appropriée. Faire

65. Voir supra.

66. Sur lesquels, voir C. André, Droit pénal spécial, $2^{\mathrm{e}}$ éd., Paris, Dalloz, 2013, nº 210, p. 173-175.

67. Le proxénétisme par fourniture de locaux est passible de 10 ans d'emprisonnement et de 750 ooo euros d'amende.

68. Cour EDH, 11 décembre 2007, Tremblay c. France.

69. Loi no 2016-444 du 13 avril 2016, art. 5. En cas de torture ou d'actes de barbarie, de violences, de violences ayant entraîné une mutilation ou une infirmité, de violences mortelles, d'agressions sexuelles, ou de viol commis sur une personne qui se livre à la prostitution pendant l'exercice de son activité, cette nouvelle catégorie de victime est constitutive d'une circonstance aggravante aux termes de l'article 11 de la loi du 13 avril 2016 venant modifier plusieurs articles du Code pénal par l'insertion de la formule suivante: «Sur une personne qui se livre à la prostitution, y compris de façon occasionnelle, si les faits sont commis dans l'exercice de cette activité». Voir Code pénal, $5^{\circ}$ quarter des articles $222-3$, 222-8, 222-10, 222-12, 222-13, l'article 222-24, $13^{\circ}$ et l'article 222-28, $9^{\circ}$.

70. L'article 611-1 nouveau du Code pénal dispose que: «Le fait de solliciter, d'accepter ou d'obtenir des relations de nature sexuelle d'une personne qui se livre à la prostitution, y compris de façon occasionnelle, en échange d'une rémunération, d'une promesse de rémunération, de la fourniture 
référence à «l'achat d'un acte sexuel » laisse à penser qu'il s'agit d'un objet que l'on pourrait se procurer. L'incrimination du « recours à l'achat d'une prestation sexuelle auprès d'une personne qui se prostitue » aurait peut-être mieux traduit la réalité. La création de cette infraction s'oppose à la condition de validité du contrat posée à l'article 1128 nouveau du Code civil tenant à son « contenu licite». L'exigence d'un "contenu licite et certain $»^{71} \mathrm{du}$ contrat posée à l'article 1128 nouveau du Code civil remplace les deux conditions objectives relatives à «l'objet certain qui forme la matière de l'engagement» et la «cause licite dans l'obligation ». Si le vocabulaire employé est différent, il ne traduit pas pour autant un abandon des notions. Les fonctions qui étaient attribuées à la cause par la jurisprudence sont notamment maintenues ${ }^{72}$.

Un but contraire à l'ordre public. L'article 1162 nouveau $\mathrm{du}$ Code civil exige quant à lui que les stipulations du contrat comme son but soient conformes à l'ordre public. Derrière cette nouvelle formulation, l'on reconnaît les exigences liées à ce que l'on appelait la «cause subjective» ou «cause du contrat». Les dispositions de l'article 1162 nouveau du Code civil ainsi appliquées au contrat de prostitution étudié permettent de démontrer que, si le prostitué-prestataire s'engage à fournir un tel service au client-bénéficiaire, c'est bien parce qu'il attend une somme d'argent en contrepartie. Or, étant donné que la loi prohibe désormais l'achat d'un acte sexuel, la cause du contrat rebaptisée «but du contrat» est contraire à l'ordre public textuel car contraire à la loi. Ce contrat est aujourd'hui contraire à l'ordre public en ce qu'il vise la réalisation d'une infraction pénale. Le contrat est alors sans effet en vertu des articles $1128^{73}$ et $1162^{74}$ du Code civil. Concrètement, cela signifie qu'une personne prostituée ne peut désormais plus saisir le juge pour exiger l'exécution du contrat, alors que cela était théoriquement possible jusqu’à présent.

\section{B. Les apories du droit face au contrat de prostitution}

Droit fiscal et prostitution. Depuis un arrêt rendu par le Conseil d'État le 4 mai $1979^{75}$, une jurisprudence administrative constante estime que les revenus tirés par la personne qui se prostitue de son activité indépendante doivent être regardés comme relevant de la catégorie des bénéfices non commerciaux (BNC) ${ }^{76}$. La prostitution est ainsi reconnue comme une activité professionnelle indépendante ${ }^{77}$, ce qui est difficilement concevable eu égard au nouveau statut de victimes de la prostitution, lequel rejette implicitement toute activité économique indépendante. Les personnes qui exercent cette activité, même à titre occasionnel, doivent alors déclarer leurs revenus et demeurent soumises à l'impôt sur le revenu. C'est en cela également que le contrat de prostitution marque toute son ambivalence car, entre ombre et lumière, il produit des effets malgré sa contrariété à l'ordre public.

Concernant les autres types d'impôts, la doctrine traditionnelle du ministère de l'Économie et des Finances consiste à laisser les personnes qui se prostituent en dehors du champ de la taxe sur la valeur ajoutée (TVA) et de la taxe professionnelle ${ }^{78}$. Mais la jurisprudence illustre que tel n'est pas toujours le cas ${ }^{79}$, et là encore le droit est ambigu et contradictoire.

Les personnes qui exercent une activité prostitutionnelle sont également soumises aux cotisations de Sécurité sociale prélevées par l'Union de recouvrement des cotisations de Sécurité sociale et d'allocations familiale (URSSAF) du fait de leur activité. La position de l'administration fiscale française est vivement critiquée

d'un avantage en nature ou de la promesse d'un tel avantage est puni de l'amende prévue pour les contraventions de la $5^{\mathrm{e}}$ classe./ Les personnes physiques coupables de la contravention prévue au présent article encourent également une ou plusieurs des peines complémentaires mentionnées à l'article 131-16 et au second alinéa de l'article 131-17». Les peines complémentaires mentionnées se traduisent par des stages de sensibilisation à la lutte contre l'achat d'actes sexuels, lesquels peuvent être accomplis aux frais de l'intéressé. La récidive est constitutive d'un délit puni d'une amende de 3750 euros (art. 225-12-1, al. $1^{\text {er }}$ du Code pénal).

71. Sur lequel, voir S. Pellet, «Le contenu licite et certain du contrat», Droit et patrimoine, n 258, 2016, p. 61 sq.

72. Voir C. Grimaldi, «Les maux de la cause ne sont pas qu'une affaire de mots», Recueil Dalloz, 2015, p. 814; G. Wicker, «La suppression de la cause par le projet d'ordonnance: la chose sans le mot?», Recueil Dalloz, 2015, p. 1557.

73. Art. 1128 du Code civil: «Sont nécessaires à la validité d’un contrat: $1^{\circ}$ Le consentement des parties; $2^{\circ}$ Leur capacité de contracter; $3^{\circ}$ Un contenu licite et certain".

74. Art. 1162 du Code civil: «Le contrat ne peut déroger à l'ordre public ni par ses stipulations, ni par son but, que ce dernier ait été connu ou non par toutes les parties».

75. CE, 4 mai 1979, nº 09337.

76. Voir notamment: CE, 17 janvier 1990, nº 43499; CAA Bordeaux, 27 avril 2006, nº 02BXo1379. L'arrêt rendu a infirmé le jugement de première instance par lequel les juges avaient procédé au redressement fiscal d'une personne qui se livrait à la prostitution dans la catégorie des bénéfices industriels et commerciaux (BIC). La catégorie des BNC regroupe, aux termes de l'article 92 du Code général des impôts: «[...] les bénéfices des professions libérales, des charges et offices dont les titulaires n'ont pas la qualité de commerçants et de toutes occupations, exploitations lucratives et sources de profits ne se rattachant pas à une autre catégorie de bénéfices ou de revenus".

77. Sur la reconnaissance en droit interne de la prostitution comme une activité professionnelle par le droit fiscal, voir notamment B. Marrion, «Profession: prostitué!», in Le sexe et la norme, spéc. p. 374-379.

78. Voir D. Derycke, Les politiques publiques et la prostitution...

79. CAA Lyon, 16 février 2010, nº 08 LYo0640. Le caractère d'indépendance de l'activité étant une condition de l'assujettissement à la TVA, et la femme exerçant son activité prostitutionnelle dans un salon de massage dont elle était propriétaire, cette dernière a été soumise à la TVA. La CAA a ainsi relevé que le "chiffre d'affaires, en vertu des articles 256 et 302 ter du Code général des impôts, dépassait la limite fixée par ce dernier article et qu'elle était ainsi assujettie à la taxe sur la valeur ajoutée». 
sur ce point ${ }^{80}$, ce qui a conduit certains auteurs à affirmer que la France est un État que l'on pourrait qualifier de proxénète ${ }^{81}$. S'acquitter des cotisations de Sécurité sociale devrait permettre à la personne qui se prostitue de pouvoir bénéficier des prestations sociales. Cette affiliation est pourtant rare, car seuls certaines URSSAF l'acceptent ${ }^{82}$.

Un parcours de sortie de la prostitution. Le fisc et l'URSSAF, de par les impôts et les cotisations exigés, calculés en fonctions des revenus de l'année antérieure, peuvent constituer un sérieux frein au désir de sortir de la prostitution. Ce problème était au cœur de l'arrêt Tremblay ${ }^{83}$, la décision rendue n'allant pas dans le sens d'une réinsertion des personnes qui se prostituent. Mais la loi du 13 avril 2016 et son décret d'application du 28 octobre 2016 contiennent plusieurs dispositions relatives à l'accompagnement des victimes de la prostitution en créant notamment un parcours de sortie de la prostitution et d'insertion sociale et professionnelle ${ }^{84}$ qui peut donner lieu au versement d'une aide financière par un fond de prévention de la prostitution et à l'accompagnement des personnes prostituées ${ }^{85}$. Ce fond sera constitué par les crédits de l'État affectés au soutient de toute initiative visant à la sensibilisation des populations aux effets de la prostitution sur la santé et à la réduction des risques sanitaires, à la prévention de l'entrée dans la prostitution et à l'insertion des personnes prostituées, ainsi que par les recettes provenant de la confiscation des produits du proxénétisme. L'engagement de la personne dans ce parcours, autorisé par le préfet ${ }^{86}$, permettra de présumer ces personnes comme répondant aux conditions de gêne ou d'indigence, ce qui peut conduire l'administration fiscale à accorder des remises totales ou partielles d'impôts ${ }^{87}$.

Si l'on retient que le contrat de prostitution ne porte pas sur le corps de la personne mais sur une prestation de service sexuel, la réalisation de cette dernière se révèle incontestablement consubstantielle au corps humain. Contrairement à ce que soutient une partie de la doctrine, ce n'est pas la réalisation d'une obligation de faire classique (laquelle n'existe d'ailleurs plus qu'implicitement), car la spécificité de la mise à disposition du corps est double. En effet, cette prestation de service s'exécute par un corps-à-corps, puisque le créancier de l'obligation met son corps à contribution de façon équivalente à celui du débiteur de l'obligation. De plus, le résultat de cette prestation de service est inhérent au corps de la personne qui l'exécute. Il n'y a pas, contrairement à la force de travail par exemple, d'extériorité du résultat de la prestation qui puisse se détacher de la personne qui l'a réalisée.

C'est pourquoi il apparaît important que ce contrat soit inséré dans la théorie générale des contrats portant sur le corps humain dont nous proposons la création, afin que ce contrat ne soit justement pas, dans l'esprit de nos contemporains, détaché du corps humain et, partant, banalisé. L'article 19 de la loi du 13 avril 2016 va

80. Dans l'affaire Tremblay c. France, par un jugement du 17 décembre 1998, le tribunal des affaires de Sécurité sociale de Paris avait annulé pour illégalité les contraintes de recouvrement des cotisations des années antérieures que l'URSAFF réclamait à une femme qui exerçait une activité prostitutionnelle. Les juges avaient été particulièrement audacieux dans leur motivation en relevant que «l'État, un et indivisible, ne peut par le biais du Ministère de la Justice faire tomber sous le coup de la loi pénale quiconque appréhende les subsides d'une personne se livrant à la prostitution et par le biais du Ministère des Finances ou d'organismes officiels, tel que l'URSAFF, appréhender lui-même lesdits revenus pour des raisons fiscales évidentes [...]. [...] le tribunal, qui a une mission de justice, ne peut que stigmatiser cette compromission étatique». Ces éléments de procédure sont rappelés par la Cour européenne des droits de l'homme dans l'arrêt Tremblay c. France, \$10.

81. Voir notamment J.-M. Larralde, «La France, État proxénète?», Revue trimestrielle des droits de l'homme, nº 77, janvier 2009, p. 195.

82. Voir G. François-Dainville, «La prostitution et le droit de la Sécurité sociale: la question de l’affiliation», Droit social, 2005 , p. 888 sq.; C. Geslot, «Prostitution, dignité... Par ici la monnaie!», Recueil Dalloz, 2008, p. 1292 sq.

83. La requérante avait saisi la Cour européenne en soutenant que les sommes réclamées par l'URSAFF étaient si élevées que cela l'obligeait à continuer de se prostituer pour pouvoir s'en acquitter, ce qui constituait un traitement inhumain ou dégradant au sens de l'article 3 de la Convention, et un travail forcé au sens de l'article $4, \$ 2$, du même texte. La Cour européenne a néanmoins décidé que les cotisations réclamées par l'URSAFF ne constituaient ni un traitement inhumain ou dégradant, ni un travail forcé, car la requérante pouvait très bien s'acquitter de ses cotisations au moyen d'autres activités.

84. Loi du 13 avril 2016, art. 5; art. L. 121-9 du Code de l'action sociale et des familles. Le décret d'application n $2016-1467$ du 28 octobre 2016 prévoit que toute association déclarée depuis au moins 3 ans et ayant pour principal objet de venir en aide aux personnes prostituées, mais également aux femmes victimes de violences ou plus généralement aux personnes en difficulté, peut demander un agrément lui permettant de participer à l'élaboration et à la mise en œuvre de ce parcours. L'agrément, délivré par le préfet, est d'une durée de 3 ans renouvelable. Une commission départementale de lutte contre la prostitution, le proxénétisme et la traite des êtres humains aux fins d'exploitation sexuelle est également instituée dans chaque département. Elle doit notamment rendre un avis sur tous les dossiers relatifs à la mise en place ou au renouvellement du parcours de sortie de la prostitution, lesquels lui sont soumis par les associations agréées ayant procédé à leur instruction.

85. Loi du 13 avril 2016, art. 7. Suivant l'article $1^{\mathrm{er}}$ du décret du 28 octobre 2016 , une personne engagée dans un parcours de sortie de la prostitution et d'insertion sociale et professionnelle pourra également bénéficier: d'un logement dans le respect des conditions réglementaires d'accès à un logement locatif social, d'un accueil en logement-foyer ou d'un hébergement adapté à sa situation; d'un accompagnement visant à faciliter l'accès aux soins, sur le plan physique et psychologique, et aux droits; d'actions d'insertion sociale, visant à favoriser la socialisation, l'autonomie des personnes dans leur vie quotidienne et l'élaboration d'un projet d'insertion professionnelle. La décision du préfet de département d'autoriser ou de renouveler le parcours de sortie permet aussi la délivrance aux personnes étrangères d'une autorisation provisoire de séjour d'une durée minimale de six mois ouvrant droit à l'exercice d'une activité professionnelle, dans les conditions prévues à l'article L. 316-1-1 du Code de l'entrée et du séjour des étrangers et du droit d'asile.

86. Selon l'article $1^{\text {er }}$ du décret du 28 octobre 2016 relatif au parcours de sortie de la prostitution, instituant l'article R. 121-12-10 du Code de l'action sociale et des familles, ce parcours peut être autorisé pour une période de 6 mois renouvelable, dans la limite de 2 ans.

87. Art. L. $2471^{\circ}$ du Livre des procédures fiscales. 
d'ailleurs dans ce sens, avec la mise en place, à l'attention des élèves, de séances d'information sur «les réalités de la prostitution et les dangers de la marchandisation du corps ", précisant que ces séances auront pour but de contribuer à «l'apprentissage du respect dû au corps humain».

En conséquence, étant donné la récente position adoptée par le législateur qui a créé le statut de victimes de la prostitution et prohibé l'achat d'actes sexuels, et dans l'objectif d'une lecture cohérente, le contrat de prostitution, en tant que contrat portant sur le corps humain, devrait figurer, à l'instar de la gestation pour autrui, parmi les contrats contraires à l'ordre public; car garder à l'esprit que cette activité est singulière, tant dans sa réalisation que dans les conséquences physiologiques, psychologiques et sociales qu'elle induit, c'est déjà contribuer à faire un pas pour la sauvegarde de la dignité humaine. 